\title{
DETERMINAÇÃO DO PERÍODO REPRODUTIVO E DO TAMANHO DE MATURAÇÃO FƯNCIONAL DOS OVÁRIOS DE Ucides cordatus (LINNAEUS, 1763) (BRACHYURA, OCYPODIDAE) NA BAÍA DE VITÓRIA, ESPÍRITO SANTO
}

\author{
Flávia Duarte Ferraz Sampaio ${ }^{1}$, Tania Mara Simões do Carmo ${ }^{2}$, Antonio Ostrensky, \\ Gisele Castilho, Thayzi de Oliveira Zeni
}

$\begin{array}{ll}1 & \text { UFPR } \\ 2 & \text { UFES }\end{array}$

Endereço para correspondência: Flávia Duarte Ferraz Sampaio: fdfsampaio@hotmail.com

\begin{abstract}
RESUMO: O caranguejo-uçá Ucides cordatus (Linnaeus, 1763), é um importante recurso pesqueiro na costa Brasileira, possuindo legislação específica para sua proteção. Este estudo teve como objetivo a análise do período reprodutivo e o tamanho de primeira maturação de fêmeas de $U$. cordatus, como subsídio à legislação de proteção à espécie no Estado do Espírito Santo. Os animais foram coletados mensalmente, em uma área do manguezal da Baía de Vitória (20 $10^{\circ} 20^{\prime \prime} \mathrm{S}$ e $40^{\circ}$ 18' 30" W), no município da Serra, ES, durante o período de um ano (outubro de 1998 a setembro de 1999). As coletas foram realizadas manualmente com o auxílio de caranguejeiros. No laboratório, procedeu-se à biometria dos caranguejos. As 280 fêmeas coletadas foram mensuradas, pesadas e a presença de ovos nos pleópodes, registrada. A determinação do período reprodutivo foi feita através da análise da Relação Gonadossomática, do Fator de Condição Alométrico e da presença de fêmeas ovígeras. No ano analisado, o período reprodutivo estendeu-se de dezembro a abril. $\mathrm{O}$ tamanho mínimo de primeira maturação das fêmeas foi de $43,2 \mathrm{~mm}$ de largura e $32,7 \mathrm{~mm}$ de comprimento de carapaça.
\end{abstract}

Palavras-chave: relação gonadossomática; reprodução; caranguejo-uçá.

\section{REPRODUCTIVE PERIOD AND OVARIAN SIZE MATURITY IN Ucides cordatus (LINNAEUS, 1973) (BRACHYURA, OCYPODIDAE) IN THE VITORIA BAY, ESPIRITO SANTO}

\begin{abstract}
The mangrove-land crab, Ucides cordatus (Linnaeus,1763), regionally known as "caranguejo uçá", is an important fishing resource in the Brazilian coast and possesses specific protection laws. This study analyzes the reproductive cycle and the size at maturity of the $U$. cordatus females in order subsidize laws aiming the protection of the species in the State of Espírito Santo. The animals were sampled monthly in the VitóriaBay mangrove $\left(20^{\circ} 14^{\prime} 20^{\prime \prime} \mathrm{S}\right.$ and $\left.40^{\circ} 18^{\prime} 30^{\prime \prime} \mathrm{W}\right)$ in Serra, Espírito Santo, from October 1998 to September 1999. Specimens were captured by hand with the help of crab collectors. In the laboratory, 280 females were measured, weighed, and the presence of eggs in the pleopods was registered. The reproductive period was determined through the analysis of the gonadossomatic index, allometric condition factor and the presence of ovigerous females. During the period of analysis, the reproductive cycle spanned from December to April. The minimum size of females at first maturity presented carapace $43.2 \mathrm{~mm}$ wide and 32.7 long.
\end{abstract}

Key Words: Gonadossomatic index; reproduction; mangrove-land crab. 


\section{INTRODUÇÃO}

Ucides cordatus (Linnaeus, 1763) (Brachyura, Ocypodidae) é um dos principais recursos pesqueiros do Brasil (Gläser, 2003). Também desempenha um importante papel ecológico nesses ecossistemas, sendo considerada uma espécie-chave na ciclagem de nutrientes e na oxigenação do solo (Wolff et al., 2000).

Durante uma fase especifica do ciclo reprodutivo, conhecida como andada ou carnaval, os animais saem das suas tocas e passam a vagar pelos manguezais (Alcântara-Filho, 1978; Costa; 1979; Nascimento, 1993). A andada dura de três a quatro dias, ocorrendo entre os meses de dezembro a maio e apresentando padrões distintos de variação latitudinal (Alcântara-Filho, 1978; Costa; 1979). Nesta fase os caranguejos ficam altamente expostos à predação por outros animais e se tornam também mais vulneráveis à ação dos catadores.

O estudo da dinâmica reprodutiva do $U$. cordatus é de extrema importância para o estabelecimento da legislação de ordenamento pesqueiro, pois orienta a tomada de decisões mais adequadas ao manejo desse recurso (Wunderlich et al., 2008).

A regulamentação do processo de captura de caranguejos e, consequentemente, as principais iniciativas de conservação desse recurso através do ordenamento pesqueiro, são feitas pelo Instituto Brasileiro do Meio Ambiente e dos Recursos Naturais Renováveis (IBAMA). As portarias publicadas regionalmente visam resguardar principalmente 0 período reprodutivo e o tamanho mínimo de captura. No entanto, para que esse tipo de regulamentação possa ser eficiente, é indispensável a geração de dados científicos sobre a dinâmica reprodutiva da espécie (Wunderlich et al., 2008).
Trabalhos enfocando estes aspectos foram realizados no Piauí (Ivo et al., 1999), Ceará (Alves, 1975; Alcântara-Filho, 1978; Costa, 1979), Rio Grande do Norte (Vasconcelos et al., 1999), Pernambuco (Botelho et al., 1999), Rio de Janeiro (Souza, 1999), Paraná (Nakamura, 1979; Dalabona et al., 2005; Dalabona e Loyola-Silva, 2005; Castilho, 2010), e Santa Catarina (Branco, 1993; Wunderlich et al., 2008). Entretanto, não há dados disponíveis para o Estado do Espírito Santo.

O objetivo do presente trabalho foi determinar o período reprodutivo e o tamanho de primeira maturação das fêmeas de $U$. cordatus no manguezal da Baía de Vitória.

\section{MATERIAL E MÉTODOS}

O trabalho foi realizado na região norte da Baía de Vitória, no município da Serra, ES, de coordenadas geográficas 200 14' S e 40o 18' W. A área total amostrada foi de aproximadamente 13,2 ha.

As coletas ocorreram mensalmente no período de outubro de 1998 a setembro de 1999. Foram realizadas em quatro transeções distantes $100 \mathrm{~m}$ uma da outra que se estenderam perpendicularmente desde o esteiro até a região de apicum. A captura dos animais foi feita manualmente por catadores profissionais de caranguejos, sendo capturadas todas as fêmeas cujas tocas encontravam-se abertas no momento da coleta.

De cada animal, foram registrados a largura da carapaça (Wid) (medida ao nível do segundo par de pereiópodes, de um lado ao outro), o comprimento (Lt) (distância entre a margem anterior até margem posterior da carapaça), o peso total (wt) e o peso dos ovários (wg). Também foi checada a presença da massa de ovos aderidos aos pleópodos modificados. 

cordatus (linnaeus, 1763) (Brachyura, ocypodidae) na Baila de Vitória, Esplitito Santo.

Para determinação da época de reprodução foram analisadas as variações da Relação Gonadossomática (RGS), do Fator de Condição Alométrico $(\Delta K)$ e feito o registro mensal de fêmeas ovígeras ao longo de todo o período de estudo. Também foi monitorada em campo a ocorrência de andada para acasalamento e liberação de larvas, no manguezal da Baía de Vitória.

A RGS foi calculada a partir da expressão de Vazzoler (1996), modificada:

$$
R G S=w g /(w t .100)
$$

Sendo:

$w g=$ peso das gônadas

$\mathrm{wt}=$ peso total do animal

O Fator de Condição Alométrico foi calculado a partir da expressão de Vazzoler (1996):

Sendo:

$$
\Delta \mathrm{K}=\mathrm{wg} / \mathrm{wid}^{\wedge} \mathrm{b}
$$

$w g=$ peso das gônadas

wid = largura da carapaça

$b=$ coeficiente de regressão entre $\mathrm{wt} /$ wid.

$\mathrm{wt}=$ peso total do animal

As medidas lineares (Wid e Lt) foram feitas com um paquímetro e o peso $(\mathrm{Wt})$ registrado em uma balança eletrônica digital. A carapaça foi seccionada dorsalmente para que os ovários fossem removidos e pesados em balança analítica de precisão.

A análise das classes de tamanho e a confecção da curva de maturidade sexual foi feita com base em Vazzoler (1996). Para isso, determinouse o intervalo existente entre a menor e a maior largura de carapaça das fêmeas adultas coletadas. $\mathrm{O}$ resultado foi dividido por 20, obtendo-se dessa maneira as classes de tamanho.
As classes de tamanho também foram analisadas a partir da fórmula proposta por Sturges (Christmann, 1978):

Sendo:

$$
\mathrm{Nc}=1+(3,3 \times \log n)
$$

$\mathrm{Nc}=$ número de classes a ser utilizado $\mathrm{n}$ = número de indivíduos

Para efeito de melhor discussão dos dados, calculou-se também o comprimento mínimo de $1^{\text {a }}$ maturação, através da mesma metodologia.

Parâmetros ambientais foram coletados conforme 0 seguinte: os dados relativos a pluviosidade foram obtidos junto à Empresa Capixaba de Pesquisa, Assistência Técnica e Extensão Rural (EMCAPER); mensalmente foi medida a temperatura do ar, com um termômetro e a salinidade da água no interior de 20 tocas escolhidas aleatoriamente, com um refratômetro de salinidade

\section{RESULTADOS}

Ao todo foram coletadas 280 fêmeas de $U$. cordatus, havendo uma prevalência $(46,78 \%)$ de animais entre 50 e $65 \mathrm{~mm}$ de largura de carapaça.

A variação dos valores médios da relação gonadossomática (RGS) e do fator de condição alométrico $(\Delta \mathrm{K})$ ao longo do ano mostraram a ocorrência de picos em dezembro e março (Figura $1 \mathrm{~A}$ e 1B).
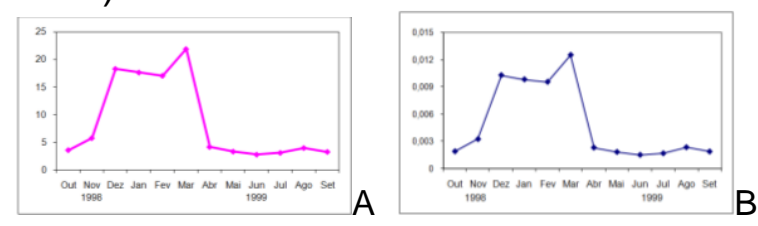

Figura 1 - A - Variação mensal dos valores médios da Relação Gonadossomática (RGS) de fêmeas de $U$. cordatus ; B - Variação mensal dos valores médios do Fator de Condição $(\Delta K)$ de fêmeas de $U$. cordatus.

Em janeiro, durante o primeiro pico reprodutivo, ocorreu a primeira 
andada para acasalamento. Foi também quando foram registrados os maiores valores da RGS e do $\Delta K$ (Figura 1). Fêmeas ovígeras foram coletadas nos meses de janeiro, fevereiro e março, sendo que a freqüência relativa foi respectivamente $60 \%, 13,79 \%$ e $53,57 \%$.

Nos meses em que a freqüência relativa de fêmeas ovígeras foi maior, janeiro e março, observaram-se os dois picos reprodutivos, o que permite inferir que a desova ocorre alguns dias após a cópula. No mês de fevereiro, a freqüência relativa de fêmeas ovígeras foi bem menor, quando comparada aos outros meses.

Foram registrados eventos de andadas entre os dias 18 e 23 de janeiro, 17 e 21 de fevereiro e 20 e 23 de março, todos durante a lua nova.

O mês de fevereiro foi caracterizado pela liberação de larvas, no qual se observou uma queda dos indicadores (RGS e $\Delta K$ ), seguida da recuperação dos ovários, culminando com 0 segundo pico reprodutivo, ocorrido em março.

Não foi observada relação direta entre a RGS e a largura da carapaça da fêmea (Figura 2).

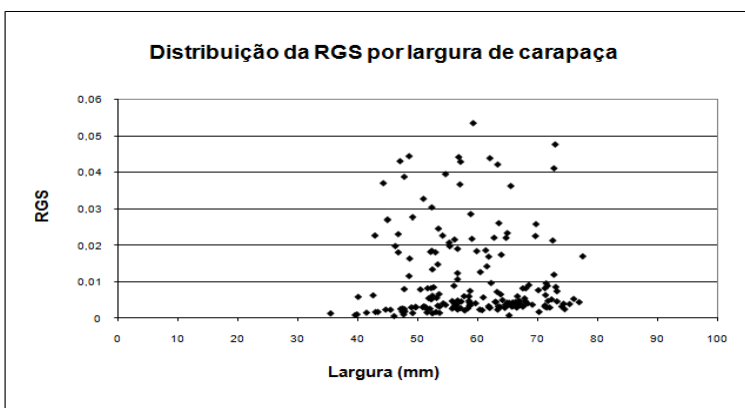

Figura 2 - Relação entre a RGS e a largura da carapaça de fêmeas de $U$. cordatus.

Observou-se que em janeiro as massas de ovos estavam mais pesadas, em fevereiro houve uma redução consistente no peso e, em março, nova variação positiva (Figura 3).

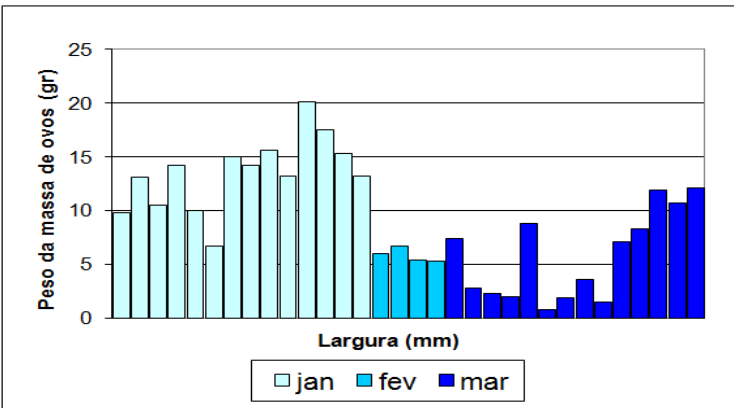

Figura 3: Distribuição de fêmeas ovígeras em relação ao peso da massa de ovos.

O período reprodutivo não apresentou relação com o período de maior pluviosidade, mas coincidiu com 0 de maiores temperaturas (Figuras $4 \mathrm{e}$ 5). Em fevereiro, quando a freqüência de fêmeas ovígeras foi mais baixa, observou-se um aumento na salinidade da água no interior das tocas (Figura 6).

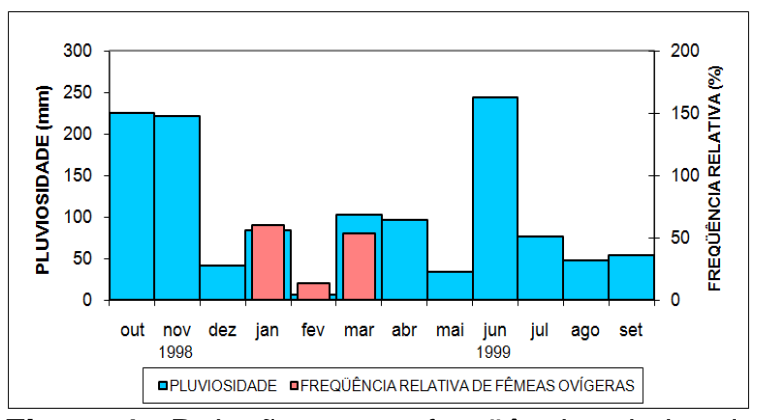

Figura 4 - Relação entre a freqüência relativa de fêmeas ovígeras de $U$. cordatus e a média mensal de pluviosidade.

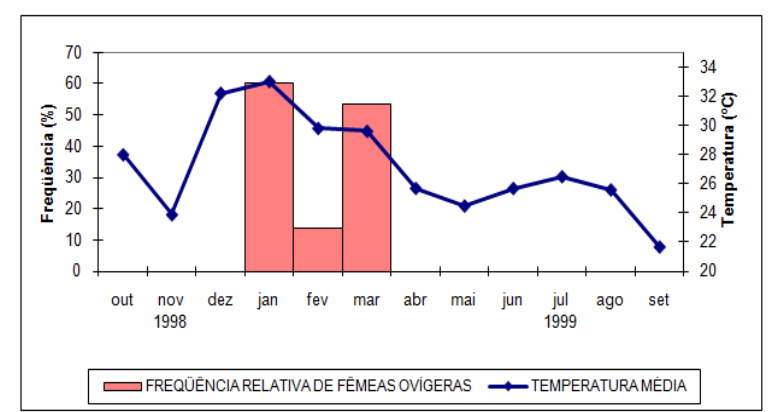

Figura 5 - Relação entre a freqüência relativa de fêmeas ovígeras de $U$. cordatus e a temperatura média da área de estudo. 
Determinação do período reprodutivo e do tamanho de maturação funcional dos ovários de ucides Cordatus (IInnaeus, 1763) (Brachyura, ocypodidae) na Baía de Vitória, Espirito Santo.

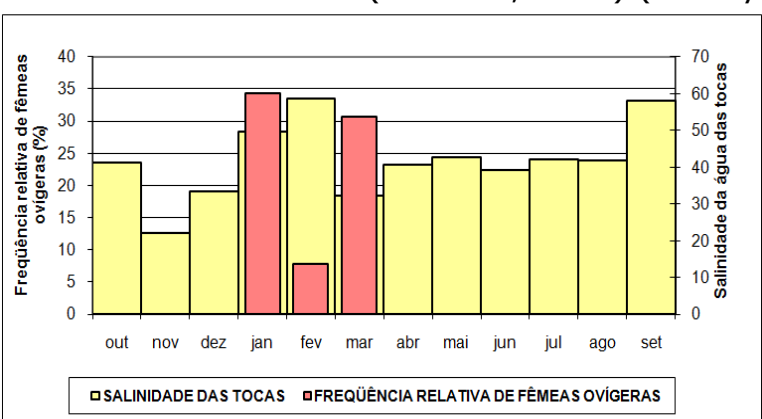

Figura 6: Relação entre a freqüência relativa de fêmeas ovígeras de $U$. cordatus e a salinidade da água no interior das tocas

Utilizando a metodologia proposta por Vazzoler (1996), foram definidas 20 classes de tamanho (Tabela 1) para as fêmeas e o tamanho mínimo de primeira maturação foi estimado em 42,7 mm de largura de carapaça (Figura 7). Já a aplicação da fórmula de Sturges permitiu a separação em nove classes (Tabela 2) e a estimação do tamanho mínimo de primeira maturação em um valor muito próximo a este, 43,2 $\mathrm{mm}$ de largura de carapaça (Figura 8).

Tabela 1: Distribuição de freqüência de largura de carapaça de fêmeas de $U$. cordatus por classes de tamanho, segundo a metodologia de Vazzoler (1996).

\begin{tabular}{|c|c|c|}
\hline Classes & JovensAdultos & $\%$ de Adultos Adultosaadultos \\
\hline $17,30 \dashv 20,312$ & 20 & 0 \\
\hline $20,31 \dashv 23,321$ & 0 & 0 \\
\hline $23,32 \nmid 26,332$ & 0 & 0 \\
\hline $26,33 \dashv 29,342$ & 0 & 0 \\
\hline $29,34 \nmid 32,354$ & 0 & 0 \\
\hline $32,35-35,362$ & 0 & 0 \\
\hline $35,36 \dashv 38,377$ & 0 & 0 \\
\hline $38,37 \dashv 41,385$ & 1 & 16,66 \\
\hline $41,38 \dashv 44,396$ & 7 & 53,85 \\
\hline $44,39 \dashv 47,403$ & 13 & 81,25 \\
\hline $47,40 \nmid 50,413$ & 22 & 88 \\
\hline $50,41-53,420$ & 30 & 100 \\
\hline $53,42 \dashv 56,430$ & 25 & 100 \\
\hline $56,43 \dashv 59,440$ & 27 & 100 \\
\hline $59,44-62,450$ & 19 & 100 \\
\hline $62,45-65,460$ & 29 & 100 \\
\hline $65,46 \dashv 68,470$ & 29 & 100 \\
\hline $68,47 \dashv 71,480$ & 19 & 100 \\
\hline $71,48 \dashv 74,490$ & 17 & 100 \\
\hline $74,49-77,500$ & 5 & 100 \\
\hline
\end{tabular}

Tabela 2: Distribuição de freqüência de largura de carapaça de fêmeas de $U$. cordatus por classes de tamanho, segundo a fórmula de Sturges (Christmann, 1978).

\begin{tabular}{|c|c|c|}
\hline Classes & Adultos & $\%$ de Adultos Adultosadultos \\
\hline $17,30 \nmid 23,983$ & 0 & 0 \\
\hline $23,98-30,665$ & 0 & 0 \\
\hline $30,66-37,3411$ & 0 & 0 \\
\hline $37,34-44,0212$ & 6 & 33.33 \\
\hline $44,02 \nmid 50,706$ & 37 & 86.04 \\
\hline $50,70 \dashv 57,380$ & 67 & 100 \\
\hline $57,38 \dashv 64,060$ & 51 & 100 \\
\hline $64,06 \dashv 70,740$ & 52 & 100 \\
\hline $70,74-77,500$ & 30 & 100 \\
\hline
\end{tabular}

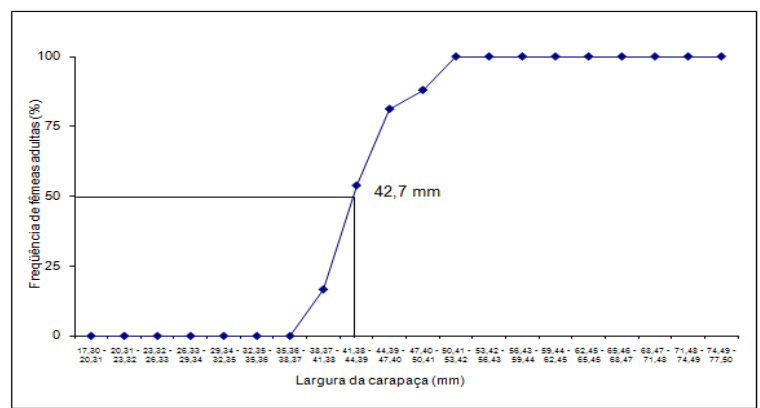

Figura 7: Freqüência acumulada das larguras da carapaça de fêmeas $U$. cordatus considerando a maturidade funcional dos ovários, de acordo com a metodologia proposta por Vazzoler (1996).

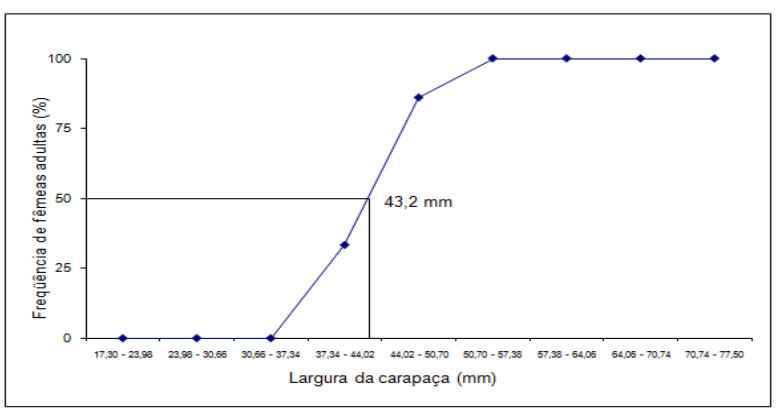

Figura 8: Freqüência acumulada das larguras de carapaça de fêmeas de $U$. cordatus considerando a maturidade funcional dos ovários, de acordo com a fórmula de Sturges (Christmann, 1978).

O comprimento mínimo de primeira maturação segundo a fórmula de Sturges (Christmann, 1978) foi de 32,7mm (Figura 9). 


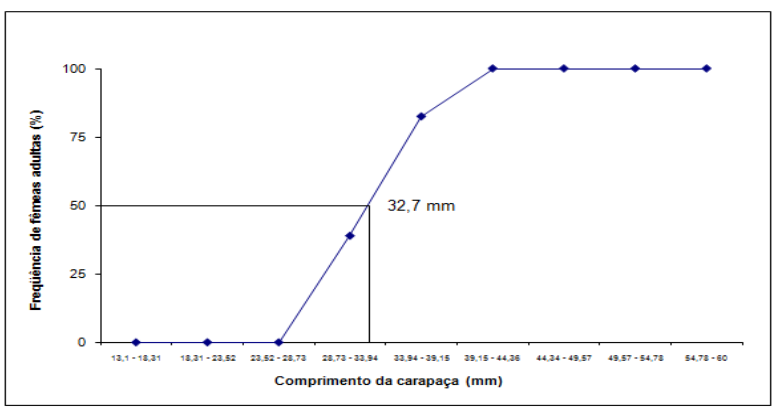

Figura 9: Freqüência acumulada dos comprimentos de carapaça de fêmeas de $U$. cordatus considerando a maturidade funcional dos ovários, de acordo com a fórmula de Sturges (Christmann, 1978).

\section{DISCUSSÃO}

O período reprodutivo de $U$. cordatus na região estudada estendeuse de dezembro a abril. Este período coincidiu, com pequenas variações, com os registrados para estados da região Nordeste (Alves, 1975; Alcântara-Filho, 1978; Costa, 1979; Castro, 1986; Botelho et al., 1999; Ivo et al., 1999). $\mathrm{Na}$ região Sul, o mesmo período foi registrado no Paraná (Nakamura, 1979; Dalabona et al., 2005; Dalabona e Loyola-Silva, 2005; Castilho, 2010) e Santa Catarina (Branco, 1993; Wunderlich et al., 2008). Na região Sudeste, no Rio de Janeiro, foram identificadas fêmeas de $U$. cordatus no estádio maduro com maior freqüência na primavera e verão, indicando ser este o período de reprodução, enquanto o estádio desovado ocorreu no outono (Souza, 1999). Estes períodos sazonais são coincidentes com os meses encontrados no presente trabalho.

Os dados indicaram a inexistência de relação entre RGS e a largura da carapaça da fêmea de U. cordatus, o que evidencia que a RGS está relacionada à época do ano e ao estágio de vida (adulto ou juvenil) e não ao tamanho do animal.

Durante o período de estudo, a primeira andada para acasalamento ocorreu em janeiro e as andadas seguintes, para acasalamento e para eclosão e liberação de larvas, ocorreram em fevereiro e março. Já em abril foi registrada andada apenas para eclosão e liberação de larvas. De acordo com Góes et al. (2000), no ano 2000 o período de andada foi maior, estendendo-se de janeiro a maio.

A metodologia aqui empregada se mostrou bastante eficiente para registrar os principais eventos reprodutivos da espécie. $O$ peso das gônadas foi mínimo até que os animais atingissem o tamanho estimado de primeira maturação (aproximadamente $42 \mathrm{~mm}$ de largura de carapaça). Como era esperado, foram registradas reduções significativas do peso das gônadas nos períodos posteriores às desovas e seu aumento até atingir o pico seguinte. Esse padrão reprodutivo, segundo Vazzoler (1996), caracteriza uma desova do tipo parcelada ou múltipla. A existência de dois picos nos índices indicativos do período reprodutivo, RGS e $\Delta \mathrm{K}$, constatada em $U$. cordatus, também sinalizam para a ocorrência de desovas parceladas.

Tais conclusões diferem das de outros autores, que não classificaram a desova de U. cordatus como múltipla. Alves (1975) descreveu a desova como total. Entretanto, o autor observou que de janeiro a maio ocorriam fêmeas com as gônadas vazias, juntamente com fêmeas em vários estádios de maturação, inclusive fêmeas maduras. Costa (1979), ao analisar a ocorrência de fêmeas ovígeras, observou que a reprodução no Ceará apresentava dois períodos mais intensos, sendo 0 primeiro em janeiro e o segundo em março-abril, coincidindo com os dois picos de desova observados no presente trabalho. No Rio Grande do Norte, fêmeas ovígeras foram encontradas entre os meses de janeiro a maio, com maior concentração entre janeiro e março (Vasconcelos et al., 1999). 

cordatus (IInnaeus, 1763) (Brachyura, ocypodidae) na Baía de Vitória, Esplrito santo.

Reigada e Negreiros-Fransozo (1999) observaram que fêmeas de muitas espécies de braquiúros podem gerar mais de uma desova após uma única cópula, e isto é possível devido ao fato de apresentarem espermateca, na qual armazenam os espermatóforos. Desta maneira, a grande variação do desenvolvimento gonadal que ocorre nas fêmeas ovígeras, pode ser entendida pela estratégia da desova múltipla.

Ciclos reprodutivos sazonais são usualmente iniciados por mudanças ambientais, tais como aumento de luminosidade e temperatura (MelvilleSmith, 1987). No presente trabalho, tentou-se verificar se tal situação ocorria, sendo que no manguezal da Baía de Vitória a reprodução de $U$. cordatus aconteceu nos meses mais quentes, mas não nos mais chuvosos. No Pará, os eventos de eclosão larval de $U$. cordatus foram restritos à estação chuvosa (Diele, 2000), enquanto no Ceará, Alcântara-Filho (1978) ressaltou que janeiro foi 0 mês de maior ocorrência de eclosão e que tais eventos ocorreram na estação chuvosa. As andadas para acasalamento e liberação de larvas de $U$. cordatus apresentaram sincronismo lunar, estando relacionadas às marés máximas e mínimas de sizígia. No presente estudo, as andadas ocorreram nos períodos de lua nova. No ano posterior, a primeira andada ocorreu durante a lua nova $e$ as andadas subsequentes, registradas em fevereiro a maio, ocorreram durante a lua cheia (Góes, et al., 2000). Freire (1998) concluiu que no Paraná a liberação de larvas apresentou também o mesmo padrão lunar, com um pico de zoea I nos manguezais durante as luas cheia e nova.

Góes, et al. (2000) registraram a presença de larvas dentro das tocas, mas, no presente trabalho, observou-se que a liberação de larvas de $U$. cordatus ocorreu principalmente nos canais do manguezal.

A determinação do tamanho de primeira maturação sexual baseia-se em estudos morfológicos externos e estudos morfofuncionais por meio de observações macro e microscópicas da forma e da condição de desenvolvimento e funcionamento das gônadas (Costa, 1995; Reigada e Negreiros-Fransozo, 1999).

Em alguns braquiúros, é possível a determinação da maturação morfológica dos indivíduos jovens e adultos, diferenciando-os pelo formato e a aderência do abdome ao esternito torácico (Melo, 1996). Em U. cordatus, é impossível fazer tal distinção, pois mesmo os menores exemplares jovens capturados (17,3 $\mathrm{mm}$ a menor fêmea) possuíam o formato do abdome similar ao dos adultos e não selado ao esterno. Segundo Reigada e NegreirosFransozo, (1999), o tamanho em que o caranguejo atinge a maturidade morfológica muitas vezes não está em sincronia com o funcionamento gonadal, sendo muito discutida a questão da existência ou não de uma sincronia de maturidade morfológica com a gonadal. Esta afirmação é reforçada por Pinheiro e Fransozo (1998), que afirmam não existir sincronismo em algumas espécies entre o desenvolvimento de caracteres secundários e a maturidade fisiológica. Segundo os pesquisadores, um exemplo ocorre com machos de Arenaeus cribrarius (Decapoda, Brachyura, Portunidae), para os quais a maturidade fisiológica precede a maturidade morfológica.

Em regiões distintas e sob diferentes condições, uma mesma espécie de Brachyura pode atingir a maturidade sexual com tamanhos menores, sendo possível encontrar fêmeas ovígeras com tamanhos variados conforme 0 local (Hartnoll,1974). 
Esta afirmação pode ser confirmada para U. cordatus, uma vez que o comprimento médio da carapaça de primeira maturação sexual varia entre populações de latitudes diferentes, ao longo do litoral brasileiro. Em alguns estados do Nordeste foram encontrados os seguintes comprimentos médios: $45,0 \mathrm{~mm}$ no Piauí com variação entre $21,4 \mathrm{~mm}$ e $62,0 \mathrm{~mm}$ (Ivo et al., 1999); $37,2 \mathrm{~mm}$ no Ceará com variação entre $35,1 \mathrm{~mm}$ e $48,7 \mathrm{~mm}$ (Alves, 1975); 41,5 $\mathrm{mm}$ no Rio Grande do Norte com variação entre $17,0 \mathrm{~mm}$ e $64,0 \mathrm{~mm}$ (Vasconcelos et al., 1999); em Pernambuco, no estuário do Rio Formoso, o comprimento foi de $40,5 \mathrm{~mm}$ com variação entre $14,0 \mathrm{~mm}$ e $58,6 \mathrm{~mm}$ e no estuário do rio llhetas foi de 38,0 $\mathrm{mm}$ com variação entre $9,5 \mathrm{~mm}$ e 57,0 $\mathrm{mm}$ (Botelho et al., 1999). No Rio de Janeiro a largura média da carapaça de primeira maturação sexual foi em torno de $47,2 \mathrm{~mm}$ com variação entre 38,6 $\mathrm{mm}$ e $68,5 \mathrm{~mm}$ (Souza, 1999). No sul, no Estado do Paraná, o comprimento foi de $30,7 \mathrm{~mm}$ com variação entre 30,0 $\mathrm{mm}$ e $54,0 \mathrm{~mm}$ (Nakamura, 1979) e a largura foi de $43 \mathrm{~mm}$ (Dalabona, 2005).

Desta forma, o tamanho mínimo de primeira maturação gonadal de fêmeas de U. cordatus no manguezal da Baía de Vitória, 43,2 mm de largura (variação de largura entre 17,3 e $77,5 \mathrm{~mm}$ ) e 32,7 $\mathrm{mm}$ de comprimento (variação de comprimento entre 13,1 e $60 \mathrm{~mm}$ ) de carapaça, foi próximo ao valor encontrado em outras regiões do país.

As primeiras portarias do IBAMA que incluíam o estado do Espírito Santo com o objetivo de conservar o $U$. cordatus ( $\mathrm{n}^{\circ}$ 35-N de 19 de abril de 1998 e no 104 de 27 de julho de 1998) não contemplavam o período reprodutivo da espécie, o que motivou pesquisas a fim de solucionar o problema, subsidiando a reformulação das portarias com base em dados científicos. No decorrer deste período, várias alterações foram feitas, sendo que o Espírito Santo foi 0 primeiro estado a ter portarias específicas para proteger a andada. Atualmente o tema é regulado pela Instrução Normativa Interministerial $\mathrm{n}^{\circ}$ $1 / 2011$ que abrange os estados os seguintes estados: Pará, Amapá, Alagoas, Bahia, Ceará, Maranhão, Paraíba, Piauí, Pernambuco, Rio Grande do Norte, Sergipe e Espírito Santo. O documento determinou os períodos de defeso da andada com base nas maiores amplitudes das marés e nas ocorrências das luas nova e cheia.

\section{CONCLUSÃO}

A Relação Gonadossomática (RGS) e o Fator de Condição $(\Delta \mathrm{K})$ se mostraram indicadores eficientes dos eventos reprodutivos de $U$. cordatus, permitindo a conclusão de que o período reprodutivo da espécie no Espírito Santo, não diferiu significativamente do observado em outras regiões do País.

A reprodução de $U$. cordatus foi do tipo parcelada, com dois picos de desovas, em janeiro e em março, não sendo observado sincronismo entre a maturidade morfológica e a maturidade gonadal em fêmeas de $U$. cordatus.

O tamanho mínimo de maturação gonadal de fêmeas de $U$. cordatus, por volta de $42-43 \mathrm{~mm}$, indica que indivíduos muito menores que os de tamanho permitido para captura, que devem ter pelo menos $6 \mathrm{~cm}$ de carapaça, estão aptos à reprodução.

Os dados aqui obtidos devem ser levados em consideração pelos órgãos de ordenamento pesqueiro e nos programas de manejo e conservação dos estoques de $U$. cordatus.

95

\section{Agradecimentos}

Ao Fundo de Apoio à Ciência e Tecnologia do Município de Vitória - 
Determinação do período reprodutivo e do tamanho de maturação funcional dos ovários de ucides cordatus (IInnaeus, 1763) (Brachyura, ocypodidae) na Baía de Vitória, Espirito santo.

FACITEC/PMV, pelo financiamento parcial da pesquisa.

\section{REFERÊNCIAS}

ALCÂNTARA-FILHO, P. Contribuição ao estudo da biologia e ecologia do caranguejo uçá, Ucides cordatus cordatus (Linnaeus, 1763) (CRUSTACEA, DECAPODA, BRACHYURA), no manguezal do rio Ceará. Arquivos de Ciências do Mar, v.18, n.1/2, p. 1-41, 1978.

ALVES, M.I.M. Sobre a reprodução do caranguejo uçá, Ucides cordatus cordatus (Linnaeus), em mangues do Estado do Ceará. Arquivos de Ciências do Mar, v.15, n. 2, p. 85 - 91, 1975.

BOTELHO, E.R.O., DIAS, A.F.; IVO, C.T.C. Estudo sobre a biologia do caranguejo-uçá, Ucides cordatus cordatus (Linnaeus, 1763), capturado nos estuários dos rios Formoso (Rio Formoso) e llhetas (Tamandaré), no Estado de Pernambuco. Boletim Técnico Científico, CEPENE, v.7, n.1, p. 9-52, 1999.

BRANCO, J.O. Aspectos bioecológicos do caranguejo Ucides cordatus (LINNAEUS, 1763) (CRUSTACEA, DECAPODA) do manguezal do Itacorubi, Santa Catarina, BR. Arquivos de Biologia e Tecnologia, v. 36, n. 1, p 133-148, 1993.

CASTILHO, G. G.; OSTRENSKY, A.; PIE, M. R. et al. (in press) Morphology of the female reproductive system and reproductive cycle of the mangrove land crab Ucides cordatus (L.) in the Baía de Antonina, Paraná, Brazil. Acta Zoologica (Stockholm). 2010.

CASTRO, A. C. L. Aspectos bio-ecológicos do caranguejo-uçá, Ucides cordatus cordatus (LINNAEUS, 1763), no Estuário do Rio dos Cachorros e Estreito do Coqueiro, São Luís MA. Boletim do Laboratório Hidrobiologia, v. 7, p 7-26, 1986.

CHRISTMANN, R.U. Estatística Aplicada. 2.ed. São Paulo : Edgar Blücher, 1978. 454p.

COSTA, R.S. Bioecologia do caranguejo-uçá, Ucides cordatus (Linnaeus, 1763) - Crustáceo,
Decápode - no Nordeste Brasileiro. Boletim da Sociedade Cearense de Agronomia, v. 20, p. 1-74, 1979.

COSTA, T.M. Ciclo reprodutivo de Callinectes danae SMITH, 1869 (CRUSTACEA, DECAPODA, PORTUNIDAE) na região de Ubatuba (SP). 1995. Botucatu. 94 f. Dissertação (Mestrado em Ciências Biológicas) - Curso de Pós Graduação em Ciências Biológicas Zoologia, Universidade Estadual Paulista Júlio de Mesquita Filho.

DALABONA, G.; LOYOLA-SILVA, J. Período reprodutivo de Ucides cordatus (Linnaeus) (Brachyura, Ocypodidae) na Baía das Laranjeiras, sul do Brasil. Acta Biológica Paranaense, v.34, p.115-126. 2005.

DALABONA, G.; LOYOLA-SILVA, J.; PINHEIRO, M.A.A. 2005. Size at morphological maturity of Ucides cordatus (Linnaeus, 1763) (Brachyura, Ocypodidae) in the Laranjeiras Bay, Southern Brazil. Brazilian archives of biology and technology, n.48, v.1, p.139-145. 2005.

DIELE, K. Population ecology of the exploited mangrove crab Ucides cordatus, Caeté estuary, North Brazil. In: MANGROVE 2000 SUSTENTABILIDADE DE ESTUÁRIOS E MANGUEZAIS: DESAFIOS E PERSPECTIVAS, 2000, Recife. Anais... Recife: UFRPE, 2000, p. 69.

FREIRE, A.S. Dispersão larval do caranguejo do mangue Ucides cordatus (L.1763) em manguezais da Baía de Paranaguá, Paraná. 1998. São Paulo, 68f. Tese (Doutorado em Oceanografia) - Curso de Pós-graduação em Oceanografia, Instituto Oceanográfico da Universidade de São Paulo.

GLÄSER, M. Interrelations between mangrove ecosystem, local economy and social sustainability in Caeté Estuary, North Brazil, Wetlands Ecology and Management, v.11, p.265-272. 2003.

GÓES, P.; SAMPAIO, F.D.F.; CARMO, T.M.S. et al. Comportamento e período reprodutivos do caranguejo do mangue Ucides cordatus. In: SIMPÓSIO DE ECOSSISTEMAS BRASILEIROS: CONSERVAÇÃO, 5, 2000, 
Vitória. Anais... São Paulo: ACIESP, 2000. v.2, p.335-348.

HARTNOLL, R.G. Variation em growth pattern between some secondary sexual characters in crabs (Decapoda, Brachyura). Crustaceana, n.27, p.130-136, 1974.

IVO C.T.C; DIAS, A.F.; MOTA, R.I. Estudo sobre a biologia do caranguejo-uçá, Ucides cordatus cordatus (Linnaeus, 1763), capturado no delta do rio Parnaíba, Estado do Piauí. Boletim Técnico Científico, CEPENE, v.7, n.1, p.53-84, 1999.

MELO, G.A.S. Manual de identificação dos Brachyura (caranguejos e siris) do litoral brasileiro. São Paulo:Pleiade/FAPESP, 1996.

MELVILLE-SMITH, R. The reproductive biology of Geryon maritae (Decapoda, Brachyura) of South West Africa/Namibia. Crustaceana, v.53, n.3, p.259-275, 1987.

NAKAMURA, I. T. Sobre a fenologia de Ucides cordatus (L., 1763), Crustacea - Brachyura, da Baía de Paranaguá. 1979. Curitiba, $71 f$. Dissertação (Mestrado em Ciências Biológicas) - Curso de Pós-graduação em Zoologia, Universidade Federal do Paraná.

NASCIMENTO, S.A. Biologia do caranguejo-uçá (Ucides cordatus). Aracaju: Adema, 1993. 48p.

PINHEIRO, M.A.A.; FRANSOZO, A. Sexual maturity of the speckled swimming crab Arenaeus cribrarius (Lamarck, 1818) (Decapoda, Brachyura, Portunidae), in the Ubatuba littoral, São Paulo state, Brazil. Crustaceana, v.71, n.4, p.434-452, 1998.

REIGADA, A.L.D.; NEGREIROS-FRANSOZO, M.L. Maturidade sexual em Hepatus pudibundus (DECAPODA, BRACHYURA, CALAPPIDAE). Iheringia, Série Zoológica, Porto Alegre, v. ${ }^{-} 96$ p.159-164, 1999.

SOUZA, E. P. Distribuição, aspectos reprodutivos e morfométricos do caranguejouçá, Ucides cordatus (LINNAEUS, 1763) no manguezal de Itacuruçá-Coroa Grande, Baía de Sepetiba, RJ. 1999. Dissertação (Mestrado em
Biologia Animal) - Universidade Federal Rural do Rio de Janeiro.

VASCONCELOS, E.M.S.; VASCONCELOS, J.A.; IVO, C.T.C. Estudo sobre a biologia do caranguejo-uçá, Ucides cordatus cordatus (Linnaeus, 1763), capturado no estuário do rio Curimatau (Canguaretama) no Estado do Rio Grande do Norte. Boletim Técnico Científico. CEPENE, v.7, n.1, p.85-116, 1999.

VAZZOLER, A.E.A.M. Biologia da reprodu 90 de peixes teleósteos: teoria e prática. Maringa : EDUEM, São Paulo: SBI, 1996. 169 p.

WOLFF, M.; KOCH, V.; ISAAC, V. A trophic flow model of the Caeté mangrove estuary (North Brazil) with considerations for the sustainable use of its resources. Estuarine, Coastal and Shelf Science, v.50, p.789-803. 2000.

WUNDERLICH, A.C.; PINHEIRO, M.A.A.; RODRIGUES, A.M.T. Biologia do caranguejouçá, Ucides cordatus (Crustacea: Decapoda: Brachyura), na Baía da Babitonga, Santa Catarina, Brasil. Revista Brasileira de Zoologia, v.25, n.2, p. 188-198. 2008. 